\title{
Fitting Curves and Surfaces with Monotonic and Non- Monotonic Four Parameter Equations
}

\author{
John Mandel* \\ National Bureau of Standards, Washington, DC 20234
}

August 6, 1980

\begin{abstract}
This is a series of three papers in which methods are presented, with instructions on computational details, on the empirical fitting of tabulated data. Part I deals with fitting functions of a single argument; Part II with functions of two arguments; and Part III with functions of three or more arguments.

Key words: Curve fitting; empirical fitting; surface fitting.
\end{abstract}

\section{Part I. Fitting Functions of a Single Argument}

\section{Introduction}

The ready availability of calculators and computers has had a profound effect on the use of tables of com. plicated functions. For example, in statistical work one may be required in a specific computer program, to call on critical values of the $F$ distribution for specified degrees of freedom in the numerator and the denominator, at specified levels of significance. It is totally impractical to store the entire $F$ table in the memory of the computer, but it is entirely feasible to let the computer calculate the required value by a suitable approximation formula.

Similar situations occur for physical or chemical properties that are tabulated as functions of temperature, pressure, wave-length, etc.

The object of this paper is to present a widely applicable procedure for finding empirical representations of tabulated values. The tabulated values are of course assumed to be derived from reasonably smooth functions of the arguments. The approximation formulas are expected to generate values that are practically interchangeable with the corresponding tabulated values.

We will present the procedure in three parts. Part $I$ is concerned with the empirical fitting of curves, i.e., functions of a single argument. Part II deals with functions of two arguments. The case of functions of more than two arguments is discussed in Part III.

Part I consists of two sections. In section 1, we deal with monotonic functions, and in section 2, with functions that have a single maximum or a single minimum.

\section{Monotonic functions}

\subsection{The general formula}

Polynomials, which are widely used for empirical fitting, have well-known shortcomings for the fitting of monotonic functions: they often have undesirable maxima, minima, and inflection points. The formula we propose in this section applies to monotonic functions, with or without a single inflection point in the range over which the curve is fitted. The formula is

$$
y_{i}=y_{0}+A\left(x_{i}-x_{0}\right)\left|x_{i}-x_{o}\right|^{B-1} ;-\infty<x<+\infty
$$

-National Measurement Laboratory. 
where $\left(x_{i}, y_{i}\right)$ are the coordinates of the points to be fitted by a monotone curve; and $x_{o}, y_{o}, A$, and $B$ are four parameters the values of which have to be estimated. Note that $B$ need not be an integer.

A simpler formula would be:

$$
y=y_{0}+A\left(x-x_{0}\right)^{B}
$$

but this formula presents difficulties for $x<x_{o}$, because of the ambiguity of defining $\left(x-x_{o}\right)^{B}$ for negative values of $x-x_{0}$. Equation (1) is totally free of this shortcoming. We will refer to eq (1) as the "four parameter equation" for monotonic functions and denote it by the symbol MFP.

\subsection{Nature of the MFP function}

Table 1 presents the properties of the MFP function in diagrammatic form. The function is defined for the entire range $x=-\infty$ to $x=+\infty$. Note that for $B<0$, the curve is discontinuous at $x_{o}$ and consists essentially of two branches, each of which is free of points of inflection. Note also that in this case $(B<0)$, the curve has finite asymptotes, equal to $y_{o}$, both at $+\infty$ and $-\infty$.

TABLE 1. MFP: Nature of Function

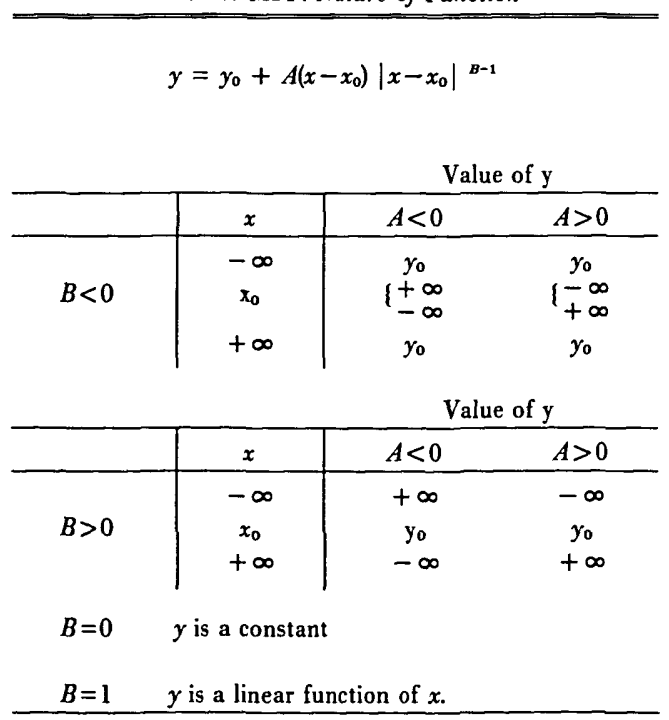

For $B>0, y$ becomes infinite both at $x=-\infty$ and $x=+\infty$ and has a point of inflection at $x_{0}$.

For $B=1$, the curve becomes a straight line and for $B=0$, it becomes a constant. It is worth noting that the curve is increasing when $A B>_{0}$ and decreasing when $A B<0$.

It is apparent that by choosing appropriate portions of the curve, with the proper parameter values, great flexibility is available, and it may therefore be expected that the curve will provide good fits for many sets of empirical data representing monotone functions with no more than one point of inflection. This does not mean, of course, that it will provide satisfactory fits for all sets of monotone data.

Figures (la) through (le) show some examples of curves that were generated by eq (1). The four parameters are given for each case. The figures demonstrate the flexibility that can be achieved through the use of this general formula.

\subsection{Method of fitting}

The procedure we use for finding the four parameters consists of two steps: (a) finding initial values for $x_{o}$ and $B$; (b) iterating, using the Gauss-Newton procedure, to improve these estimates. 


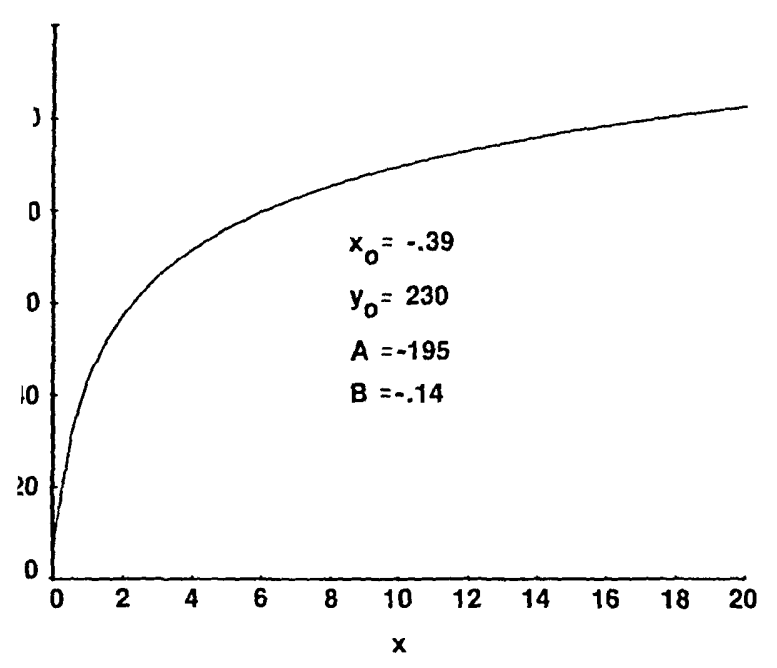

Figure 1-a.

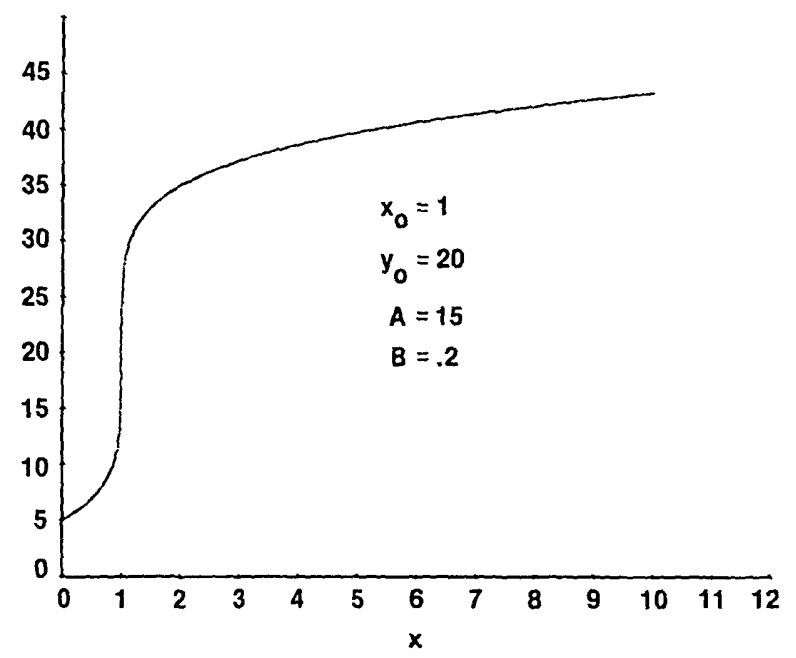

Figure I-c.

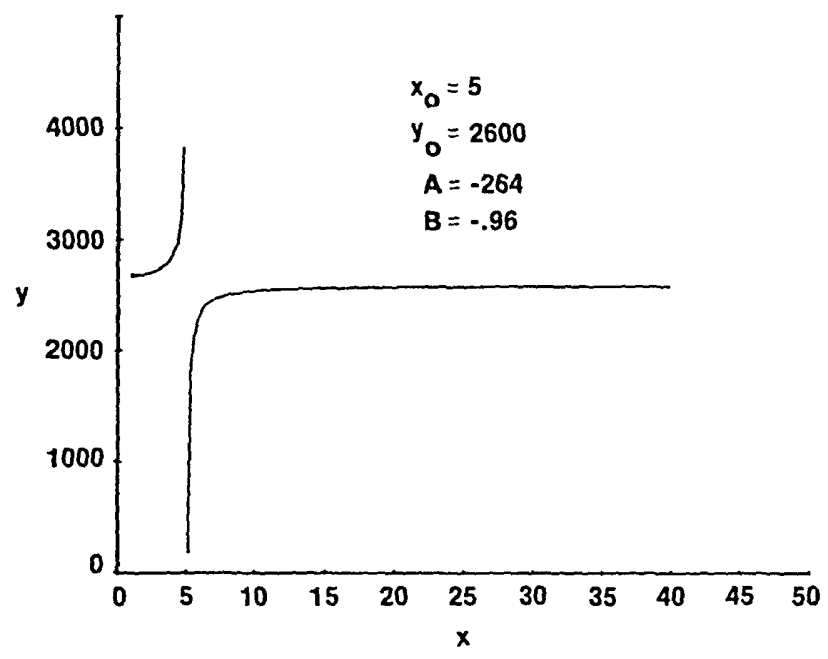

Ficure 1-e. 
Both steps are presented with a great deal of detail, in spite of the fact that standard procedures can be found in the literature for non-linear fitting. The reason for this is the intent to make this paper essentially self-contained.

Once $x_{o}$ and $B$ are known, define

$$
z_{i} \equiv\left(x_{i}-x_{0}\right)\left|x_{i}-x_{0}\right|^{B-1}
$$

Then, eq (1) becomes:

$$
y_{i}=y_{0}+A z_{i}
$$

which is the equation of a straight line in $y_{i}$ versus $z_{i}$, for which the intercept $y_{o}$ and the slope A are readily estimated by linear regression.

The variable $z_{i}$ can be regarded as a reexpression of $x_{i}$ in a transformed scale. The new scale must be such that $z_{i}$ is linearly related to $y_{i}$. For the purpose of comparing different pairs of $\left(x_{o}, B\right)$ in achieving a good fit, a convenient measure is therefore the correlation coefficient between $z_{i}$ and $y_{i}$. We will use this measure throughout the paper with the understanding that it is merely a comparative measure for the adequacy of a $\left(x_{0}, B\right)$ pair of values, and that we are not concerned here with the statistical properties of this measure.

\subsection{Initial values for $x_{0}$ and $B$}

Differentiating $\mathrm{y}$ with respect to $\mathrm{x}$, in $\mathrm{Eq}(1)$, gives the relation:

$$
\frac{d y}{d x} \equiv y_{x}^{\prime}=A B\left|x-x_{o}\right|^{B-1}
$$

Dividing eq (1) by eq (5) yields:

$$
\frac{y-y_{0}}{y_{x}^{\prime}}=\frac{x-x_{0}}{B}
$$

or.

$$
y=y_{0}+\frac{1}{B}\left(x y_{x}\right)-\frac{x_{0}}{B}\left(y_{x}\right)
$$

Now $x$ and $y$ are given for $N$ points of the curve, and $y_{x}^{\prime}$ can be approximately calculated for the midpoints of the intervals between successive $x$-values. The value of $y$ can also be estimated approximately at these midpoints. This yields $N-1$ sets of values $x, y$, and $y_{x}^{\prime}$ from which $x y_{x}^{\prime}$ can be calculated. A multiple linear regression of $y$ on $x y_{x}^{\prime}$ and on $y_{x}^{\prime}$, allowing for the constant term $y_{0}$, then gives estimates of the coefficients $y_{0}, \frac{1}{B}$ and $\frac{x_{0}}{B}$. We ignore the first and use the two others to estimate $x_{0}$ and $B$.

\subsection{Illustrative example for finding initial values of $x_{0}$ and $B$}

An important statistical application of empirical curve and surface fitting is to represent standard statistical tables by formulas that can be used for ready interpolation or for incorporation into computer programs. Our first example is the two-tail 5 percent critical value of Student's $t$, for values of $v$, the degrees of freedom, ranging from 2 to $\infty$. The data used for the fit consist of 20 selected pairs of $\left(\nu, t_{c}\right)$, where $t_{c}$ is the critical value in question [2]. The data are given in table 2 . We substituted 10,000 for $v=\infty$.

The calculations for the initial values estimation are shown in table 3. The midpoints of $x$ and $y$ are denoted $x_{m}$ and $y_{m}$. The derivative $y_{x}^{\prime}$ is approximated by $\Delta y / \Delta x$ (column 5). For example: 
TABLE 2. Two-Tail 5 percent Critical Values of Student's $t$ Statistic

\begin{tabular}{|c|c|}
\hline Degrees of Freedom $(\nu)$ & Student's t \\
\hline $2.0 \ldots \ldots \ldots \ldots \ldots$ & 4.3027 \\
\hline $3 \ldots \ldots \ldots \ldots$ & 3.1825 \\
\hline $4 \ldots \ldots \ldots \ldots$ & 2.7764 \\
\hline $5 \ldots \ldots \ldots \ldots$ & 2.5706 \\
\hline $6 \ldots \ldots \ldots \ldots$ & 2.4469 \\
\hline $7 \ldots \ldots \ldots \ldots$ & 2.3646 \\
\hline $8 \ldots \ldots \ldots \ldots$ & 2.3060 \\
\hline $9 \ldots \ldots \ldots \ldots$ & 2.2622 \\
\hline $10 \ldots \ldots \ldots \ldots \ldots$ & 2.2281 \\
\hline $12 \ldots \ldots \ldots \ldots \ldots$ & 2.1788 \\
\hline $14 \ldots \ldots \ldots \ldots$ & 2.1448 \\
\hline $16 \ldots \ldots \ldots \ldots$ & 2.1199 \\
\hline $18 \ldots \ldots \ldots \ldots$ & 2.1009 \\
\hline $20 \ldots \ldots \ldots \ldots \ldots$ & 2.0860 \\
\hline $25 \ldots \ldots \ldots \ldots \ldots$ & 2.0595 \\
\hline $30 \ldots \ldots \ldots \ldots$ & 2.0423 \\
\hline $40 \ldots \ldots \ldots \ldots$ & 2.0211 \\
\hline $60 \ldots \ldots \ldots \ldots$ & 2.0003 \\
\hline $120 \ldots \ldots \ldots \ldots \ldots$ & 1.9799 \\
\hline $10000 \ldots \ldots \ldots \ldots \ldots$ & 1.9600 \\
\hline
\end{tabular}

TABLE 3. Calculations for Initial Values of $x_{0}$ and $B$. (Dato in Table 2)

\begin{tabular}{|c|c|c|c|c|c|}
\hline (1) & (2) & (3) & (4) & (5) & (6) \\
\hline$x_{i}$ & $y_{i}$ & $x_{m}$ & $y_{m}$ & $\frac{\Delta y}{\Delta x}$ & $x_{m} \frac{\Delta y}{\Delta x}$ \\
\hline 2.0 & 4.3027 & 2.5 & 3.74260 & -1.120200 & -2.800500 \\
\hline 3. & 3.1825 & 3.5 & 2.97945 & -.406100 & -1.421350 \\
\hline 4. & 2.7764 & 4.5 & 2.67350 & -.205800 & -.926100 \\
\hline 5. & 2.5706 & 5.5 & 2.50875 & -.123700 & -.680350 \\
\hline 6. & 2.4469 & 6.5 & 2.40575 & -.082300 & -.534950 \\
\hline 7. & 2.3646 & 7.5 & 2.33530 & -.058600 & -.439500 \\
\hline 8. & 2.3060 & 8.5 & 2.28410 & -.043800 & -.372300 \\
\hline 9. & 2,2622 & 9.5 & 2.24515 & -.034100 & -.323950 \\
\hline 10. & 2.2281 & 11.0 & 2.20345 & -.024650 & -.271150 \\
\hline 12. & 2.1788 & 13.0 & 2.16180 & -.017000 & -.221000 \\
\hline 14. & 2.1448 & 15.0 & 2.13235 & -.012450 & -.186750 \\
\hline 16. & 2.1199 & 17.0 & 2.11040 & -.009500 & -.161500 \\
\hline 18. & 2.1009 & 19.0 & 2.09345 & -.007450 & -.141550 \\
\hline 20. & 2.0860 & 22.5 & 2.07275 & -.005300 & -.119250 \\
\hline 25. & 2.0595 & 27.5 & 2.05090 & -.003440 & -.094600 \\
\hline 30. & 2.0423 & 35.0 & 2.03170 & -.002120 & -.074200 \\
\hline 40. & 2.0211 & 50.0 & 2.01070 & -.001040 & -.052000 \\
\hline 60. & 2.0003 & 90.0 & 1.99010 & -.000340 & -.030600 \\
\hline 120. & 1.9799 & 5060.0 & 1.96995 & -.000002 & -.010191 \\
\hline 10000 . & 1.9600 & & & & \\
\hline
\end{tabular}

$$
\frac{3.1825-4.3027}{3-2}=-1.1202
$$

Finally, the value $x y_{x}^{\prime}$ is approximated by $x_{m} \frac{\Delta y}{\Delta x}$ (column 6).

The multiple linear regression is carried out by regressing column (4) on a column consisting of unity for all rows (for the coefficient $y_{0}$ ) and on columns (6) and (5). Thus, the first observational equation is:

$$
3.7426=y_{\circ}(1)+\frac{1}{B}(-2.8005)-\frac{x_{\circ}}{B}(-1.1202)
$$


The normal equations are (omitting the symmetrical elements below the diagonal in the $x^{\prime} x$ matrix):

\begin{tabular}{r|ccc}
\hline & $y_{0}$ & $\frac{1}{B}$ & $-\frac{x_{o}}{B}$ \\
\cline { 2 - 4 }-44.00215000 & 19.0 & -8.86179170 & -2.15789201 \\
-6.95277684 & & 12.14223318 & 4.10274947 \\
& & & 1.49195207 \\
\hline
\end{tabular}

Solving these 3 equations in 3 unknowns, we obtain for the second and third coefficients:

$$
\begin{aligned}
\frac{1}{B} & =-.93471932 \\
-\frac{x_{0}}{B} & =.75296356
\end{aligned}
$$

which give the estimates:

$$
\begin{aligned}
& \hat{x}_{0}=.80555 \\
& \hat{B}=-1.069839
\end{aligned}
$$

Calculating the corresponding $z_{i}$ for all $x_{i}$ by eq $(3)$ and regressing $y_{i}$ on $z_{i}$, we obtain the estimates

$$
\begin{aligned}
& \hat{y}_{o}=1.96424556 \\
& \hat{A}=2.82544745
\end{aligned}
$$

The correlation coefficient between $z_{i}$ and $y_{i}$, for the pair $\left(x_{0}=.80555, B=1.069839\right)$, is 0.999993 , indicating a quite successful fit. We now develop a Gauss-Newton iteration process to achieve, if possible, an even better fit.

\subsection{Gauss-Newton iteration to improve the fit}

The iteration is carried out on the standardized vectors of $y$ and $z$, defined as follows: ${ }^{1}$

$$
\begin{aligned}
v_{i} & \equiv \frac{y_{i}-\bar{y}}{S_{y}}, \text { where } S_{y}=\sqrt{ } \sum_{i}\left(y_{i}-\bar{y}\right)^{2} \\
t_{i} & \equiv \frac{z_{i}-\bar{z}}{S_{z}}, \text { where } S_{x}=\sqrt{ } \sum_{i}\left(z_{i}-\bar{z}\right)^{2}
\end{aligned}
$$

Define:

$$
\begin{gathered}
D_{i} \equiv v_{i}-t_{i} \text { if } \Sigma_{i} v_{i} t_{i}>0 \\
D_{i} \equiv-v_{i}-t_{i} \text { if } \Sigma_{i} v_{i} t_{i}<0
\end{gathered}
$$

Then the equations yielding the corrections for $x_{0}$ and $B$, denoted by $\Delta x_{0}$ and $\Delta B$, are:

$$
D_{i}=\frac{\partial t_{i}}{\partial x_{0}} \Delta x_{0}+\frac{\partial t_{i}}{\partial B} \Delta B
$$

' $\ell$, as defined by eq (8) should not be confused with Students's $t$ used for the illustrative example. 
The partial derivatives $\frac{\partial t_{i}}{\partial x_{o}}$ and $\frac{\partial t_{i}}{\partial B}$ are readily evaluated using eqs (3), (7), and (8). The results are:

$$
\begin{aligned}
& \frac{\partial t_{i}}{\partial x_{0}}=\frac{1}{S_{z}}\left(P_{i}-\bar{P}-E \cdot t_{i}\right) \\
& \frac{\partial t_{i}}{\partial B}=\frac{1}{S_{z}}\left(Q_{i}-\bar{Q}-F \cdot t_{i}\right)
\end{aligned}
$$

where

$$
\begin{aligned}
P_{i} & \equiv-B\left|x_{i}-x_{0}\right|^{B-1} \\
Q_{i} & \equiv z_{i} \ln \left|x_{i}-x_{0}\right|
\end{aligned}
$$

$\bar{P}$ and $\bar{Q}$ are the averages of the $P_{i}$ and the $Q_{i}$, and

$$
\begin{aligned}
& E \equiv \sum_{i} P_{i} t_{i} \\
& F \equiv \sum_{i} Q_{i} t_{i}
\end{aligned}
$$

Using these equations, $\frac{\partial t_{i}}{\partial x_{0}}$ and $\frac{\partial t_{i}}{\partial B}$ are computed for all $i$ and a multiple linear regression is carried out, in accordance with eq (11), of $D_{i}$ on $\frac{\partial t_{i}}{\partial x_{0}}$ and $\frac{\partial t_{i}}{\partial B}$. The coefficients are $\Delta x_{o}$ and $\Delta B$.

To avoid "overshooting," it is advisable to correct $x_{0}$ and $B$ by only a fraction of $\Delta x_{0}$ and $\Delta B$, say $\left(\Delta x_{0}\right) / 4$ and $(\Delta B) / 4$. Thus, the new values for $x_{0}$ and $B$ are

$$
\begin{aligned}
& \text { new } x_{0}=x_{0}+\frac{\Delta x_{0}}{4} \\
& \text { new } B=B+\frac{\Delta B}{4}
\end{aligned}
$$

Using these new values, $z_{i}$ is recalculated for all $i$ and the entire process is repeated. Iteration continues until practical convergence is reached.

Using the above equations, the entire procedure is readily programmed on the computer. The calculations are simple and rapid.

Referring to our illustrative example (table 2), and starting with the initial values $x_{o}=.80555$ and $\mathrm{B}=$ -1.069839 , we obtain after 30 iterations: $x_{o}=.836464$ and $\mathrm{B}=-1.055240$. The correlation between $z$ and $y$ is now 0.9999964 . The fitted values are correct to within 2 or 3 units in the third place. Further iterations do not improve the fit and result in only minute changes in $x_{o}$ and $B$. Table 4 lists the four parameters of the final fit and the fitted values. Figure 2 is a graph of the experimental points and of the fitted curve.

\subsection{Additional remarks}

Occasionally, the initial values for $x_{0}$ and $B$, obtained by the method described in section 4 above, are unsatisfactory and the iteration process may fail to converge. One possible remedy is to interchange $x$ and $y$ in the formula. Indeed, the basic formula $y=y_{0}+A\left(x-x_{0}\right)^{B}$ can be written:

$$
x=x_{0}+\frac{1}{A^{1 / B}}\left(y-y_{0}\right)^{1 / B}
$$

indicating the same form for expressing $x$ in terms of $y$ as vice-versa.

If this advice leads to a satisfactory fit, one can do further iterations in the original form $(y$ as a function of $x$ ), using the values of $x_{o}$ and $B$ obtained by use of the inverted formula after a few iterations. 
TABLE 4. Fit of Data of Table 2

\begin{tabular}{|c|c|c|}
\hline \multicolumn{3}{|c|}{ Equation: $y=1.963000+2.745822(\nu-.836464)^{-1.053240}$} \\
\hline Degrees of Freedom $(\nu)$ & Tabular Value & Fitted Value \\
\hline$\ldots \ldots \ldots \ldots$ & 4.3027 & 4.3032 \\
\hline$\ldots \ldots \ldots \ldots \ldots \ldots \ldots \ldots$ & 3,1825 & 3.1792 \\
\hline $4 \ldots \ldots \ldots \ldots \ldots \ldots \ldots \ldots \ldots$ & 2.7764 & 2.7775 \\
\hline $5 \ldots \ldots \ldots \ldots \ldots \ldots \ldots \ldots \ldots \ldots$ & 2.5706 & 2.5725 \\
\hline $6 \ldots \ldots \ldots \ldots \ldots \ldots \ldots \ldots \ldots$ & 2.4469 & 2.4487 \\
\hline $7 \ldots \ldots \ldots \ldots \ldots \ldots \ldots \ldots \ldots \ldots$ & 2.3646 & 2.3659 \\
\hline $8 \ldots \ldots \ldots \ldots \ldots \ldots \ldots \ldots \ldots \ldots \ldots \ldots \ldots$ & 2.3060 & 2,3068 \\
\hline $9 \ldots \ldots \ldots \ldots \ldots \ldots \ldots \ldots \ldots \ldots \ldots$ & 2.2622 & 2.2625 \\
\hline 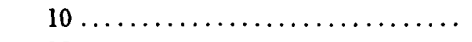 & 2.2281 & 2.2281 \\
\hline $12 \ldots \ldots \ldots \ldots \ldots \ldots \ldots \ldots \ldots \ldots$ & 2.1788 & 2.1783 \\
\hline $14 \ldots \ldots \ldots \ldots \ldots \ldots \ldots \ldots \ldots \ldots \ldots$ & 2.1448 & 2.1439 \\
\hline $16 \ldots \ldots \ldots \ldots \ldots \ldots \ldots \ldots \ldots \ldots$ & 2.1199 & 2.1188 \\
\hline $18 \ldots \ldots \ldots \ldots \ldots \ldots \ldots \ldots \ldots \ldots \ldots \ldots$ & 2.1009 & 2.0997 \\
\hline $20 \ldots \ldots \ldots \ldots \ldots \ldots \ldots \ldots \ldots \ldots$ & 2.0860 & 2.0847 \\
\hline $25 \ldots \ldots \ldots \ldots \ldots \ldots \ldots \ldots \ldots \ldots$ & 2.0595 & 2.0583 \\
\hline $30 \ldots \ldots \ldots \ldots \ldots \ldots \ldots \ldots \ldots \ldots$ & 2.0423 & 2.0411 \\
\hline $40 \ldots \ldots \ldots \ldots \ldots \ldots \ldots \ldots \ldots$ & 2.0211 & 2.0203 \\
\hline $60 \ldots \ldots \ldots \ldots \ldots \ldots \ldots \ldots \ldots \ldots$ & 2.0003 & 2.0004 \\
\hline $120 \ldots \ldots \ldots \ldots \ldots \ldots \ldots \ldots \ldots$ & 1.9799 & 1.9807 \\
\hline 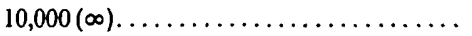 & 1.9600 & 1.9632 \\
\hline
\end{tabular}

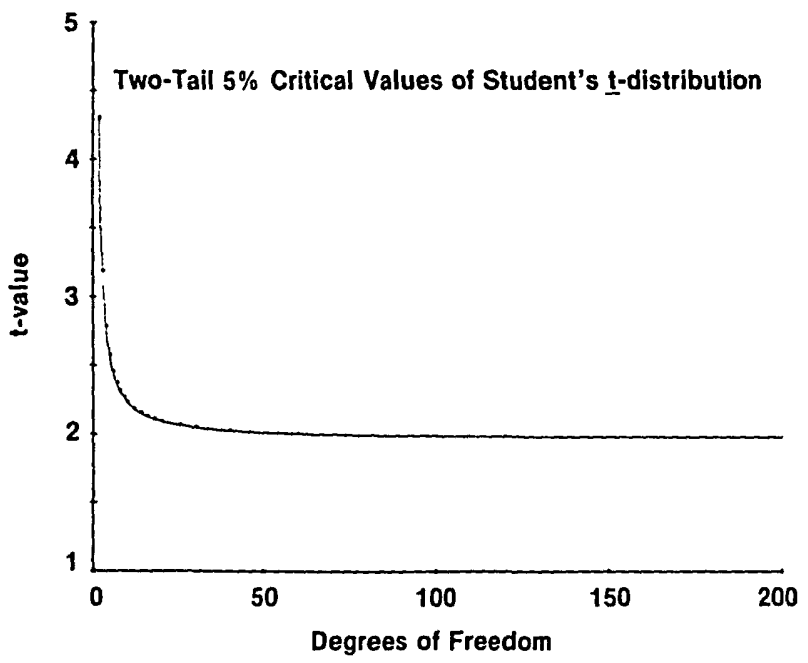

FIGURE 2. Two-tail 5 percent critical values of Student's $t$-distribution.

It is also possible to simply "guess" initial values. It happens occasionally that after an initial tendency to diverge (decreasing correlation coefficient), the process suddenly reverses itself and leads to a good fit.

As noted earlier, the general fitting equation given by (1), yields good results for many sets of monotonic data, but cannot be guaranteed to be satisfactory in all cases. 


\section{Non-Monotonic functions}

\subsection{The general formula}

We will deal with functions that present a single maximum or a single minimum in the range in which they are to be fitted. Polynomials of order 2 (quadratics) appear to be the most plausible candidates for such functions. The general equation for the quadratic is:

$$
y=a+b x+c x^{2}
$$

In practice, however, it is often found that even for functions presenting only a single maximum or a single minimum, eq (2l) provides a very poor fit indeed. For example, the five points represented by the first two columns of table 5 , when fitted by a quadratic, give the least squares fit of column 3 of that table. A fairly obvious device for improving the fit is to express the $x$ variable in a transformed scale. To be effective, the scale transformation must be non-linear. A simple example is given by the relation:

$$
y=a+b x^{\alpha}+c\left(x^{\alpha}\right)^{2}
$$

We will use a slight modification of eq (22), to allow for a frequently occurring case, namely the case in which a logarithmic transformation of $x$ is indicated.

\begin{tabular}{|c|c|c|}
\hline$x$ & $y$ & Quadratic Fit of $y^{a}$ \\
\hline 25 & .513089 & .503630 \\
\hline 10 & .551180 & .684080 \\
\hline 5 & .516202 & .329230 \\
\hline 1 & .063119 & -.104050 \\
\hline 0.5 & -.403100 & -.167547 \\
\hline
\end{tabular}

TABLE 5. A Non-Monotonic Function

a Value $\hat{y}=a+b x+c x^{2}$, resulting from unweighted least squares fit.

It can readily be shown that

$$
\ln \mathrm{x}=\lim _{\alpha \rightarrow 0} \frac{x^{\alpha}-1}{\alpha}
$$

Thus, by writing:

$$
y=a+b\left(\frac{x^{\alpha}-1}{\alpha}\right)+c\left(\frac{x^{\alpha}-1}{\alpha}\right)^{2} ; 0<x<\infty
$$

we obtain a general formula that includes the transformation of $x$ to its logarithm (for very small $\alpha$ ) as well as all cases covered by eq (22) (for any other $\alpha$ ).

Equation (24) is the formula we propose for fitting non-monotonic functions of a positive valued argument, with a single maximum or minimum. We refer to this equation as the Quadratic Four Parameter (QFP) formula. The unsolved problem is to find the value of $\alpha$ that provides the best fit by eq (24). But first we study the properties of the QFP function.

\subsection{Nature of the QFP function}

A diagrammatic presentation of the properties of the QFP function is given in Table 6.

Note that the curve can be monotonic under certain conditions, namely for $b \alpha / 2 c \geq 1$. If not monotonic, the curve is finite at one end of the range of $x$ and $\infty$ at the other end. Within this range, it passes through a local minimum or a maximum, which always occurs at $x=\left(1-\frac{b \alpha}{2 c}\right)^{1 / \alpha}$. Here again, as for the $M F P$, a great deal of flexibility is available. 
TABLE 6. QFP: Nature of Function.

$$
y=a+b\left(\frac{x^{\alpha}-1}{\alpha}\right)+c\left(\frac{x^{\alpha}-1}{\alpha}\right)^{2}
$$

(If $\frac{b \alpha}{2 c} \geq 1$, curve is monotonic)

$$
\text { Case of } \frac{b \alpha}{2 c}<1
$$

\begin{tabular}{|c|c|c|c|}
\hline \multirow[b]{2}{*}{ Value of $x$} & \multicolumn{3}{|c|}{ Value of $y$} \\
\hline & $\alpha>0$ & $\alpha<0$ & $\alpha$ very close to zero ${ }^{b}$ \\
\hline$x=0$ & $a-\frac{b}{\alpha}+\frac{c}{\alpha^{2}}$ & $\begin{array}{l}+\infty(c>0) \\
-\infty(c<0)\end{array}$ & $\begin{array}{l}+\infty(c>0) \\
-\infty(c<0)\end{array}$ \\
\hline$x=x_{m}{ }^{a}$ & $a-\frac{b^{2}}{4 c}$ & $a-\frac{b^{2}}{4 c}$ & $a-\frac{b^{2}}{4 c}$ \\
\hline$x=+\infty$ & $\begin{array}{l}+\infty(c>0) \\
-\infty(c<0)\end{array}$ & $a-\frac{b}{\alpha}+\frac{c}{\alpha^{2}}$ & $\begin{array}{l}+\infty(c>0) \\
-\infty(c<0)\end{array}$ \\
\hline
\end{tabular}

$\cdot x_{m}=\left(1-\frac{b \alpha}{2 c}\right)^{1 / \alpha} ;$ for $\alpha \approx 0, x_{m}=e^{-b / 2 c}$

bIn that case: $y=a+b \ln x+c(\ln x)^{2}$

\subsection{The fitting procedure}

Appropriate $\alpha$-values are often found in the range -2 to +2 . Since a computer program can readily be written that fits eq (24) for any given value of $\alpha$, and since the calculations are quite rapid on any programmable desk calculator, or minicomputer, a trial and error search procedure is a reasonable way to obtain an initial value for $\alpha$. It is then easy to apply a Gauss-Newton iteration process to zero in on the best value for $\alpha$.

We propose the following procedures:

1. Let

Then:

$$
u \equiv \frac{x^{\alpha}-1}{\alpha}
$$

$$
y=a+b u+c u^{2}
$$

2. Choose a small set of values of $\alpha$ between -2 and +2 . For each value of $\alpha$, calculate $u$ for each $x$, fit eq (26), and calculate the correlation coefficient $\varrho$ between $y_{i}$ and the corresponding fitted value $\hat{y}_{i}$. Also calculate:

$$
k=\frac{c}{b}
$$

With a properly-written program, step 2 should take very little time on a programmable desk calculator and much less on a minicomputer.

3. Having found a value of $\alpha$ that gives a $\varrho$-value of 0.99 or better, in absolute value, ${ }^{2}$ this $\alpha$ and the corresponding $k$ may be taken as the starting values for the iteration process, which is presently described:

4. Equation(26) is written in the form:

$$
y=a+b\left(u+k u^{2}\right)
$$

\footnotetext{
${ }^{2}$ For data of relatively low precision, it may not be possible to achieve $\varrho=.99$. We are particularly concerned, in this paper, with data of high precision, such as tabulated mathematical functions, or high precision physical or chemical data.
} 
5. Let

$$
z_{i} \equiv u_{i}+k u_{i}^{2} \quad(\mathrm{i}=1 \text { to } \mathrm{N})
$$

Then

$$
y_{i}=a+b z_{i}
$$

6. Using the same approach as for the monotonic four-parameter (MFP) curve fit, and defining $v_{i}, t_{i}$, and $D_{i}$ in the same way (eqs (7) to (10)), we obtain, as before:

$$
\begin{gathered}
\frac{\partial t_{i}}{\partial \alpha}=\frac{1}{S_{z}}\left[P_{i}-\bar{P}-E \bullet t_{i}\right] \\
\frac{\partial t_{i}}{\partial k}=\frac{1}{S_{z}}\left[Q_{i}-\bar{Q}-F \bullet t_{i}\right]
\end{gathered}
$$

However, the quantities $P$ and $Q$ are now defined by the following relations:

$$
\begin{gathered}
P_{i} \equiv \frac{1+2 k u_{i}}{\alpha}\left[\left(1+\alpha u_{i}\right) \ln x_{i}-u_{i}\right] \\
Q_{i} \equiv u_{i}^{2}
\end{gathered}
$$

The quantities $E$ and $F$ are defined, as before, by eqs (16) and(17).

In the place of eq(11), we now have:

$$
D_{i}=\frac{\partial t_{i}}{\partial \alpha} \Delta \alpha+\frac{\partial t_{i}}{\partial k} \Delta k
$$

The regression of $D_{i}$ on $\frac{\partial t_{i}}{\partial \alpha}$ and $\frac{\partial t_{i}}{\partial k}$ yields the "corrections" $\Delta \alpha$ and $\Delta k$. Again, it is advisable to use only a fraction, say one-fourth, of these quantities at each iteration.

The iteration process is continued until further impovement of the fit becomes negligible.

\subsection{An illustration}

For illustration of the QFP process, let us return to the example of table 5. Trying first a few values between -1 and 1 , one finds readily that a high correlation $(\varrho=.999263)$ is obtained for $\alpha=-0.1$, with a corresponding $k=.243550$. Using these approximations as initial estimates for the iteration process, the latter rapidly converges to a $\varrho$-value of 0.9999988 , giving the final estimates (for eq (24)):

$$
\begin{gathered}
\alpha=-.198499 \\
a=.062839 \\
b=.522744 \\
c=-.140142
\end{gathered}
$$

The effectiveness of this fitting procedure can be judged by examining table 7 , in which a number of simple transformations are compared with the one resulting from our fitting procedure. Note that the logarithmic transformation is represented by $\alpha=0$; what is actually meant is that instead of the transformation to the logarithm, eq (23) could have been applied, using a very small value of $\alpha$, such as 0.001 or 0.0001 . It is apparent that a dramatic improvement in the fit results from using the value of $\alpha$ that is given by the iteration process.

Contrary to what may be believed, the $(x, y)$ data in table 5 were not "made up." They represent one of the eigenvectors obtained in the process of fitting a table of a statistical function of three arguments by an empirical formula (see part III). The $x$-values are the values of one of the three arguments: level of significance. 
TABLE 7. Comparison of Quadratic Fits

\begin{tabular}{cc|cccr}
\hline & & \multicolumn{4}{|c}{ y fitted, using quadratic function of } \\
\hline$x$ & $\begin{array}{c}y \\
\text { observed }\end{array}$ & $x$ & $\ln x^{a}$ & $x^{-1}$ & $x^{-0.198499}$ \\
\hline 25 & 0.513089 & 0.503630 & 0.495354 & 0.560887 & 0.513351 \\
10 & .551180 & .684080 & .578775 & .530048 & .550271 \\
5 & .516202 & .329230 & .525325 & .478823 & .517023 \\
1 & .063119 & -.104050 & .014030 & .076830 & .062839 \\
0.5 & -.403100 & -.167547 & -.372926 & -.406150 & -.402995 \\
& $\alpha$ & 1 & 0 & -1 & -.198499 \\
& $\varrho$ & .89630 & .99675 & .99687 & .999999 \\
\hline
\end{tabular}

- In $\mathrm{x}$ corresponds to $\alpha=0$. See text.

\subsection{An illustration from the physical sciences and a simple stratagem}

Table 8 is taken from a tabulation of the density $d^{3}{ }^{3}$ in grams per milliliter, of ordinary water, for values of temperature ranging from -5 to $30^{\circ} \mathrm{C}[2] .^{4}$ For ease of calculations, we use the coded values $y=10^{-6}(d-$ .998). It is well known that this property has a maximum in the vicinity of $4{ }^{\circ} \mathrm{C}$. We consequently try to fit the data by the Quadratic Four Parameter (QFP) formula.

TABLE 8. Density of Ordinary Water as a Function of Temperature

\begin{tabular}{cccc}
\hline $\begin{array}{c}\text { Temperature } \\
\left({ }^{\circ} \mathrm{C}\right)\end{array}$ & $\begin{array}{c}\text { Density } \\
\text { (in } y \text {-units })\end{array}$ & $\begin{array}{c}\text { Temperature } \\
\left({ }^{\circ} \mathrm{C}\right)\end{array}$ & $\begin{array}{c}\text { Density } \\
\text { (in } y \text {-units })\end{array}$ \\
\hline-5 & 1283 & 5 & 1992 \\
-4 & 1441 & 6 & 1968 \\
-3 & 1578 & 7 & 1930 \\
-2 & 1694 & 8 & 1877 \\
-1 & 1790 & 9 & 1809 \\
0 & 1868 & 10 & 1728 \\
1 & 1927 & 15 & 1129 \\
2 & 1968 & 20 & 234 \\
3 & 1992 & 25 & -925 \\
4 & 2000 & 30 & -2322 \\
\hline
\end{tabular}

$y=10^{8}(d-0.998)$

We run into a minor difficulty in that our range of $x$-values includes negative values. This can be remedied in this case by taking as the independent variable $x=t+\beta$, where $\beta$ is chosen so as to make all $x$ - values positive. We use $\beta=10$ for our example. Thus:

$$
\begin{gathered}
x=t+10 \\
y=10^{6}(d-0.998)
\end{gathered}
$$

Trial of a few values for $\alpha$ shows that $a=0.8$ gives a fit with a correlation coefficient of 0.999967 (whereas for $a=1$, which corresponds to a "no transformation" quadratic fit, the correlation is 0.99894$)$. The corresponding $k$ value is $k=-.055752$. Application of the iteration process leads rapidly to the best parameter values:

with a correlation $\varrho=.999990$.

$$
\begin{aligned}
\alpha & =.824103 \\
a & =242.267451 \\
b & =373.749212 \\
c & =19.881303
\end{aligned}
$$

\footnotetext{
30 aroid confusion with the corelation coefficient, we use the symbol $d$, rather than the conventional $Q$ for density.

- Figures in brackets refer to literature references located at the end of each of the three parts of this paper.
} 
Table 9 presents a comparison of the simple quadratic fit (no transformation) with the QFP. For ease of evaluation, the tabulated value is the residual $y-\hat{y}$ (fitted minus observed).

The QFP can reliably be used for interpolation. Use of an empirical formula for extrapolation is of course always risky and should be done if at all, with great caution. The usefulness of the formula for interpolation is demonstrated in table 10, which shows both fits for values of $x$ not included in the fitting process (but within the range of the $x$ values used for the fit).

TABLE 9. Comparison of QFP and QLS Fits for Data of Table 6"

\begin{tabular}{c|c|c|c|c|c}
\hline \hline \multirow{2}{*}{$\begin{array}{c}\text { Temperature } \\
\left({ }^{\circ} \mathrm{C}\right)\end{array}$} & \multicolumn{2}{|c|}{ Residual } & \multirow{2}{*}{ Temperature } & \multicolumn{2}{|c}{ Residual } \\
\cline { 2 - 3 } \cline { 5 - 6 }$\left({ }^{\circ} \mathrm{C}\right)$ & QFP & QLS & & QFP & QLS \\
\hline-5 & -10 & 93 & 6 & -3 & -35 \\
-4 & -1 & 53 & 7 & -3 & -26 \\
-3 & 4 & 20 & -4 & -17 \\
-2 & 6 & -4 & 9 & -5 & -5 \\
-1 & 6 & -22 & 10 & -4 & 7 \\
0 & 5 & -35 & 15 & 2 & 77 \\
1 & 3 & -43 & 20 & 8 & 97 \\
2 & 2 & -45 & 25 & -4 & 47 \\
3 & 0 & -45 & 30 & -7 & -97 \\
4 & -1 & -41 & & & \\
\hline
\end{tabular}

a QLS = quadratic least squares fit (unweighted)

TABLE 10. Comparison of QFP and QLS Fits for Interpolation

\begin{tabular}{c|c|c|c|c|c}
\hline \hline \multirow{2}{*}{$\begin{array}{c}\text { Temperature } \\
\left({ }^{\circ} \mathrm{C}\right)\end{array}$} & \multicolumn{2}{|c|}{ Residual } & \multirow{2}{*}{ Temperature } & \multicolumn{2}{|c}{ Residual } \\
\cline { 2 - 3 } \cline { 5 - 6 }$\left({ }^{\circ} \mathrm{C}\right)$ & QLS & 21 & 9 & QFP & 94 \\
11 & -3 & 32 & 22 & 9 & 86 \\
12 & -2 & 45 & 23 & 9 & 77 \\
13 & -1 & 57 & 24 & 8 & 64 \\
14 & 1 & 68 & 26 & 5 & 26 \\
16 & 4 & 86 & 27 & 3 & 2 \\
17 & 5 & 92 & 28 & 1 & -26 \\
18 & 6 & 96 & 29 & -4 & -60 \\
19 & 7 & 97 & &
\end{tabular}

\section{Conclusion for Part I}

We have presented two formulas for the empirical fitting of functions of a single argument. The first applies to monotonic functions; the second can be used for monotonic functions under certain conditions, but its main use is for functions that have a single minimum or a single maximum. These formulas turn out to be useful also in the fitting process of functions of two or more arguments, as will be shown in Parts II and III of this paper. The fitting of the two functions is straightforward and can be readily programmed on computers and even on programmable calculators.

\section{References}

[1] Symbols, Definitions and Tables for Industrial Statistics and Quality Control, Rochester, Institute of Technology, Rochester, N.Y. (1958).

[2] Handbook of Chemistry and Physics, 56th Edition, CRC Press, Cleveland, Ohio (1975-1976). 


\section{Part ll: Fitting Functions of Two Arguments}

\section{Introduction}

Let $z_{i j}$ be a function of two arguments $x_{i}$ and $y_{j}$. The form of the function is unknown, but a set of data is available in the form of a rectangular array, in which the row "labels" are $x_{i}$ and the column "labels" $y_{j}$. The tabulated value corresponding to $x_{i}$ and $y_{j}$ is $z_{i j}$. We assume that each cell of this two-way table is "filled"; i.e.: there are no cells for which the value of $z_{i j}$ is missing.

An example will clarify matters. Table 1 is a portion of the table of 5 percent critical values of the "Studentized Range" [1]. This is a relatively short portion of the complete table but will suffice for illustration of the method, and will help to show its power.

The principle of the procedure is to first find the "Singular Value Decomposition" (SVD) [2] ${ }^{3}$ of the matrix representing the two-way table, using only the $z_{i j}$ values (but not the $x_{i}$ and the $y_{j}$ ), and then to relate the parameters of the SVD to the $x_{i}$ and the $y_{j}$, using the methods of part I of this paper.

The SVD [2] is a technique for developing the following relation:

$$
z_{i j}=\theta_{1} u_{1 i} v_{1 j}+\theta_{2} u_{2 i} v_{2 j}+\ldots+\theta_{p} u_{p i} v_{p j}
$$

or, more compactly:

$$
z_{i j}=\sum_{k=1}^{p} \theta_{k} u_{k i} v_{k j}
$$

where the $\theta_{k}$ are positive constants and the $u_{k i}$ and $v_{k j}$ are vectors such that

$$
\sum_{i} u_{k i}^{2}=\sum_{j} v_{k j}^{2}=1, \text { for all } k
$$

and

$$
\sum_{i} u_{k_{i}} u_{k^{\prime} i}=\sum_{j} v_{k^{\prime}} v_{k^{\prime} j}=0, \text { for } k \neq k^{\prime}
$$

It can be shown that $p$ is equal to the rank of the $z$-matrix, and this rank is, in turn, equal at most to the number of rows or the number of columns of the table, whichever is smaller. The $\theta_{k}$ are the square roots of the "eigenvalues" of the $z^{T} z$ matrix, where $z^{T}$ denotes the transpose of the matrix $z$, and the $v_{k j}$ are the corresponding "eigenvectors." The $u_{k i}$ are the eigenvectors of the $z z^{T}$ matrix. The $\theta$ 's are called the singular values of the matrix $z$.

\section{The SVD technique}

Algorithms for finding the SVD are readily available and a number of computer programs have been written for this purpose $[14,15,16,17]$.

In most cases, it is not necessary to consider all $p$ terms on the right hand side of eq (1). In fact, the first 2 or 3 terms are often sufficient to give an excellent approximation for the $z_{i j}$. From a practical viewpoint, it is easy to judge at what point the SVD can be terminated.

We illustrate the procedure with the data of table 1 . Denote the residuals, after fitting the first $q$ terms on the right-hand side of eq $(2)$, by $\left(d_{i j}\right)_{q}$. Thus:

$$
\left(d_{i j}\right)_{q}=z_{i j}-\sum_{k=1}^{q} \theta_{k} u_{k i} v_{k j}
$$

It can be shown that:

\footnotetext{
'The SVD procedure is intimately related to the Method of Principal Components [3]; its use, in either of these two forms for the analysis of two-way tables of data has been discussed in a number of places $[4,5,6,7,8,9,10,11,12,13]$. Figures in brackets refer to literature references, listed on page 19 .
} 


$$
\sum \sum_{i j}\left(d_{i j}\right)_{q}^{2}=\sum_{i j} z_{i j}^{2}-\sum_{k=1}^{q} \theta_{k}^{2}
$$

For the data of table 1 , we find

$$
\theta_{1}=38.034666 ; \theta_{2}=1.392726 ; \theta_{3}=.064557
$$

and

$$
\sum \sum_{i j} z_{i j}^{2}=1448.579715 .
$$

Applying eq (5), for $q=3$, we find:

$$
\sum \sum\left(d_{i j}\right)_{q=3}^{2}=0.000044
$$

This sum of squares of residuals applies to 42 observations; the residuals are not independent. Nevertheless, we can calculate an "average square residual per observation,"2 which will be equal to

$$
\frac{0.000044}{42}=1.05 \times 10^{-6}=(0.0010)^{2}
$$

Thus, after fitting the first three terms of the SVD, the residuals will be of the order of 0.001 . Since the data are given with 3 decimals, such a fit is, for most practical purposes, quite satisfactory.

Table 2 lists the $\theta$ values and the eigenvectors $u_{i}$ and $v_{j}$ for the first three terms.

TABLE 1. Five Percent Critical Values of the Studentized Range

\begin{tabular}{rcccccrrr}
\hline \hline & & & 5 & 10 & 20 & 60 & 100 \\
\hline 4 & 2 & 3 & 5.040 & 6.287 & 7.826 & 9.233 & 11.240 & 12.090 \\
8 & 3.927 & 4.041 & 4.886 & 5.918 & 6.870 & 8.248 & 8.843 \\
20 & 3.261 & 3.578 & 4.232 & 5.008 & 5.714 & 6.740 & 7.187 \\
40 & 2.950 & 3.442 & 4.039 & 4.735 & 5.358 & 6.255 & 6.645 \\
120 & 2.858 & 3.356 & 3.917 & 4.560 & 5.126 & 5.929 & 6.275 \\
$\infty$ & 2.800 & 3.314 & 3.858 & 4.474 & 5.012 & 5.764 & 6.085 \\
\hline
\end{tabular}

TABLE 2. 6-Values and Eigenvectors for Studentized Range (table 1)

\begin{tabular}{r|rrr||r|rrr}
\hline \multicolumn{1}{c|}{} & $u_{1}$ & $u_{2}$ & $u_{3}$ & \multicolumn{1}{c|}{$n$} & \multicolumn{1}{c}{$v_{1}$} & $v_{2}$ & $v_{3}$ \\
\hline 4 & .586984 & -.684908 & -.393566 & 2 & .199994 & .550013 & .514706 \\
8 & .439355 & -.132755 & .588796 & 3 & .246064 & .489632 & .178017 \\
20 & .366344 & .194757 & .446619 & 5 & .296980 & .365874 & -.212600 \\
40 & .343350 & .317316 & .117282 & 10 & .357146 & .167917 & -.490644 \\
120 & .328090 & .407791 & -.239725 & 20 & .411696 & -.032030 & -.447995 \\
$\infty$ & .320429 & .456467 & -.477211 & 60 & .489830 & -.320941 & .071843 \\
& & & 100 & .523336 & -.437783 & .459993 \\
\hline
\end{tabular}

\section{Fitting the structural parameters}

Our next task is to express the $u_{i}$ and $v_{j}$ (which we can call the structural parameters) as functions of $v$ and $n$ respectively. This is a curve fitting problem, and can be attacked by the methods developed in part I of this paper.

\footnotetext{
2No attempt is made to compute a "standard deviation of fit" with an appropriate number of degrees of freedom. The fit is purely empirical and is not based on a mathematical model; the proposed "average square residual per observation" is to be understood in that spirit.
} 
The method of section 2 of part I is used for eigenvectors that are monotonic functions of their arguments, while section 3 is applied to eigenvectors that present a single maximum or minimum. In general, the fit for the $u_{3 i}$ and $v_{3 j}$ vectors need not be as good as those for the first two sets of vectors, since the third term, having a small multiplier $\theta_{3}$, contributes only a small part to the SVD.

The entire computational procedure is summarized in Table 3 which lists the fitted vectors $u_{k i}$ and $v_{k j}$ obtained, for $k=1$ and 2, by the MFP procedure and for $k=3$, by the QFP fitting procedure. Table 4 lists the fitted values, which may be compared to the values in table 1 .

TABLE 3. Parameters of Fits

\begin{tabular}{c|rrrr}
\hline \multicolumn{1}{c|}{} & \multicolumn{3}{c}{ MFP Fits } & \multicolumn{1}{c}{$A$} \\
\hline Vector & \multicolumn{1}{c}{$x_{0}$} & \multicolumn{1}{c}{$y_{0}$} & .766066 & -.954958 \\
$u_{1}$ & .981602 & .320232 & -1.167675 & -.079057 \\
$v_{1}$ & .569248 & 1.335141 & -3.326912 & -.856578 \\
$u_{2}$ & .523691 & .459599 & 3.392462 & -.398982 \\
$v_{2}$ & -5.477500 & -.964295 & &
\end{tabular}

QFP Fits

\begin{tabular}{c|rrrr} 
Vector & \multicolumn{1}{c}{$\alpha$} & $a$ & $b$ & $c$ \\
\hline$u_{3}$ & -.776878 & -24.645495 & 47.554916 & -22.368740 \\
$v_{3}$ & -.032413 & 1.449792 & -1.543742 & .307466 \\
\hline
\end{tabular}

TABLE 4. Fitted Values for Data of Table 1

\begin{tabular}{rrrrrrrrr}
\hline$v$ & 2 & 3 & 5 & 10 & 20 & 60 & 100 \\
\hline 4 & 3.923 & 5.044 & 6.291 & 7.823 & 9.231 & 11.242 & 12.089 \\
8 & 3.261 & 4.038 & 4.889 & 5.921 & 6.867 & 8.247 & 8.848 \\
20 & 2.954 & 3.572 & 4.233 & 5.012 & 5.712 & 6.733 & 7.183 \\
40 & 2.862 & 3.435 & 4.040 & 4.739 & 5.358 & 6.253 & 6.648 \\
120 & 2.804 & 3.348 & 3.917 & 4.564 & 5.126 & 5.930 & 6.283 \\
$\infty$ & 2.776 & 3.308 & 3.859 & 4.477 & 5.007 & 5.755 & 6.081 \\
\hline
\end{tabular}

\section{Interpolation}

The total number of parameters used for the fit is 27: four for each of the two vectors in each of the three terms of the SVD, in addition to the three $\theta$-values. The actual number is less, since each $\theta$ can be incorporated into the coefficients of one of the two corresponding vectors, and further simplification is possible when the entire equation is algebraically reduced. However, this is unimportant for two reasons.

In the first place, for purposes of programming the calculations, any additional manipulation to reduce the number of parameters is unnecessary.

Secondly, and this is an important point-the often-made assertion that "it is absurd to fit a set of data with as may parameters, or almost as many parameters as there are data" can not be justified. The fact is that by fitting the 42 values of table 1 , we have obtained a formula that fits the 5 percent critical value table of the Studentized Range for all values of $v$ from 4 to infinity and all values of $n$ from 2 to 100 , using only 27 parameters. That this indeed so, can be verified by applying our fitting algorithm to any pair of $\nu$ and $n$ values $(\nu=4$ to $\infty, n=2$ to 100$)$.

The values of Table 5 illustrate this point for some selected pairs of values.

The procedure we propose is in many cases, a powerful and reliable interpolation algorithm. 
TABle 5. Examples of Fitted Values for $(v, n)$ Not Included in Fit.a

\begin{tabular}{rr|rr}
\hline & & \multicolumn{2}{|c}{ Studentized Range } \\
\hline$\nu$ & $n$ & Tabular & Fitted \\
\hline 3 & 3 & 5.910 & 5.945 \\
3 & 8 & 8.853 & 8.863 \\
6 & 50 & 13.360 & 13.352 \\
6 & 4 & 4.896 & 4.897 \\
6 & 12 & 6.789 & 6.789 \\
13 & 70 & 9.370 & 9.375 \\
13 & 2 & 3.055 & 3.059 \\
13 & 17 & 5.931 & 5.929 \\
60 & 90 & 7.667 & 7.663 \\
60 & 4 & 3.737 & 3.734 \\
60 & 50 & 5.958 & 5.956 \\
500 & 80 & 6.303 & 6.305 \\
700 & 3 & $(3.314)$ & 3.317 \\
1000 & 40 & $(5.498)$ & 5.517 \\
& 90 & $(6.020)$ & 6.042 \\
\hline
\end{tabular}

"Values in parentheses correspond to $\nu$ values not found in tables. The values in parentheses are for $\nu=\infty$.

\section{AN EXAMPle from the Physical SCIEnCES}

Table 6 lists values of the quantity $\left(n^{2}-1\right) /\left(n^{2}+2\right)$ for benzene at various values of pressure and wavelength. The values were derived from table 1 of ref. [18]. The symbol $n$ represents refractive index. The function $\left(n^{2}-1\right) /\left(n^{2}+2\right)$ is chosen, in prefernce to $n$, because of the Lorentz-Lorenz equation:

$$
\frac{n^{2}-1}{n^{2}+2} \frac{1}{D}=f(\lambda)
$$

where $D$ represents density and $\lambda$ wavelength. Since for a fixed mass, the density is a function of pressure, we can replace $D$ by $\phi(P)$, where $P$ denotes pressure. Equation (6) can then be written as:

$$
\frac{n^{2}-1}{n^{2}+2}=\phi(P) \cdot f(\lambda)
$$

According to this equation, the quantity $\left(n^{2}-1\right) /\left(n^{2}+2\right)$ is a multiplicative function of two factors, one depending on pressure only, and the other on wavelength only. It can be shown that if this is true, all but the first term of SVD of table 6 represent merely experimental error. The first term of the SVD, on the other hand represents the quantity $\phi(P) \cdot f(\lambda)$.

TABLE 6. Refractive Index of Benzene at $34.5^{\circ} \mathrm{C}$ as a Function of Pressure and Wavelength

\begin{tabular}{ccccccccc}
\hline \multicolumn{10}{c}{ Tabulated Value $=\frac{\mathrm{n}^{2}-1}{\mathrm{n}^{2}+2}$} \\
\hline \multicolumn{10}{c}{ Wavelength } \\
\hline Pressure & 6678 & 6438 & 5876 & 5086 & 5016 & 4922 & 4800 & 4678 \\
1. & 0.287528 & 0.288222 & 0.290224 & 0.294366 & 0.294869 & 0.295531 & 0.296490 & 0.297546 \\
246.2 & .293514 & .294242 & .296296 & .300494 & .301012 & .301707 & .302652 & .303743 \\
484.8 & .298497 & .299225 & .301278 & .305558 & .306072 & .306737 & .307738 & .308830 \\
757.2 & .303242 & .303964 & .306057 & .310383 & .310917 & .311597 & .312595 & .313713 \\
1107.7 & .308426 & .309152 & .311276 & .315688 & .316232 & .316921 & .317930 & .319058 \\
\hline
\end{tabular}


Eventhough our procedure makes no pretenses to anything but empirical fitting, this set of data provides us with an opportunity to examine the agreement between a physical theory (the Lorentz-Lorenz relation) and a set of experimental data.

The first three $\theta$ values of the SVD of table 6 are:

$$
\theta_{1}=1.92330513 ; \theta_{2}=0.00009801 ; \theta_{3}=0.00002689
$$

Note the very large drop from $\theta_{1}$ to $\theta_{2}$, indicating that one multiplative term in the SVD should represent the data quite well. More exactly, we find:

$$
\begin{gathered}
\sum \sum_{i j}\left(d_{i j}\right)_{1}^{2}=\sum_{i j} z_{i j}^{2}-\theta_{1}^{2} \\
=3.699102657-(1.92330513)^{2}=3.4 \times 10^{-8}
\end{gathered}
$$

The average square residual per observation is:

$$
\frac{3.4 \times 10^{-8}}{40}=8.5 \times 10^{-10}=\left(2.9 \times 10^{-5}\right)^{2}
$$

Thus, one single multiplicative term reproduces the data of table 6 to about 3 units in the 5 th place. It is easily verified that addition of a second multiplicative term fails to significantly improve this fit. The precision of a measurement of $n$ in this study is no better than 1 to 3 units in the fifth place [18]. Applying the law of propagation of errors, it is easily seen that the same statement holds for the quantity $\left(n^{2}-1\right) /\left(n^{2}+2\right)$.

We now have the model:

$$
\frac{n^{2}-1}{n^{2}+2}=\theta u_{i} v_{j}+\text { error }
$$

where $u_{i}$ is a function of pressure only, and $v_{j}$ a function of wavelength only. Thus, eq (8) is equivalent to eq (7), as required by the Lorentz-Lorenz theory.

The fit of $u_{i}$ as a function of pressure and $v_{j}$ as a function of wavelength can be accomplished by the fourparameter curve. Table 7 lists the parameters of the two curves as well as the fitted values, using eq (8). A comparison of tables 6 and 7 confirms the satisfactory quality of the fit.

TABLE 7. Fitted Values for Data of Table

\begin{tabular}{ccccccccc} 
Equation: $\frac{n^{2}-1}{n^{2}+2}=1.923305[.671861$ & $\left.-1.483692(P+1480.809979)^{-.249049}\right] \cdot\left[.333889+.091736\left(\frac{\lambda}{1000}-1.817095\right)^{-1.237982}\right]$ \\
\hline Pressure & 6678 & 6438 & 5876 & 5086 & 5016 & 4922 & 4800 & Wavelength \\
1 & 0.287552 & 0.288247 & 0.290238 & 0.294364 & 0.294840 & 0.295519 & 0.296470 & 0.297513 \\
246.2 & .293563 & .294272 & .296305 & .300517 & .301003 & .301696 & .302667 & .303732 \\
484.8 & .298467 & .299188 & .301255 & .305537 & .306032 & .306736 & .307723 & .308806 \\
757.2 & .303229 & .303961 & .306061 & .310412 & .310915 & .311630 & .312633 & .313733 \\
1107.7 & .308387 & .309133 & .311268 & .315693 & .316204 & .316931 & .317952 & .319070 \\
\hline
\end{tabular}

\section{Conclusion for Part II}

By combining the Singular Value Decomposition technique with the curve fitting procedures developed in part $\mathrm{I}$, it is possible to obtain excellent empirical fits for many sets of data in which the dependent 
(response) variable is displayed as a two-way table and the rows and columns represent levels of the two independent (regressor) variables, respectively.

The procedure consists in performing an SVD on the matrix of values of the response variable and then fitting the vectors of parameters, which are functions of the rows or of the columns, but not of both, to the corresponding regressor variables.

\section{References}

[1] Harter, H. L., Tables of Range and Studentized Range, Annals of Mathematical Statistics, 31, No. 4, 1122-1147, 1960.

[2] Rao, C. R., Linear Statistical Inference and Its Applications, (John Wiley \& Sons, N.Y., 1973).

[3] Hotelling, H., Analysis of a Complex of Statistical Variables into Principal Components, J. of Educational Psychology 24, 417-441, 498-520 (1933).

[4] Church, A., Analysis of Data when the Response is a Curve, Technometrics 8, 229-246 (1966).

[5] Gollob, H. F., A Statistical Model which combines Features of Factor Analytic and Analysis of Variance Techniques, Psychometrika 33, 73-116(1968).

[6] Jolicoeur, Pierre and J. E. Mosimann, Size and Shape Variation in the Painted Turtle. A Principal Component Analysis, Growth 24, 339-354 (1960).

[7] Mandel, John, The Partitioning of Interaction in Analysis of Variance, J. of Research of the National Bureau of Standards-B. Mathematical Sciences 73B, 309-328 (1969).

[8] Mandel, John, Distribution of Eigenvalues of Covariance Matrices of Residuals in Analysis of Variance, J. of Research of the National Bureau of Standards-B. Mathematical Sciences 73B, 149-154 (1970).

[9] Mandel, John, A New Analysis of Variance Model for Non-Additive Data, Technometrics 13, 1-18 (1971).

[10] Mandel, John, Principal Components, Analysis of Variance and Data Structure, Statistica Neerlandica 26, 119-129 (1972).

[11] Simonds, J. L., Application of Characteristic Vector Analysis to Photographic and Optical Response Data, J. Opt. Soc. Am. 53, 968-974 (1963).

[12] Snee, R. D., On the Analysis of Response Curve Data, Technometrics 14, 47-62 (1972).

[13] Wernimont, Grant, Evaluating Laboratory Performance of Spectrophotometers, Anal. Chem. 39, 554-562 (1967).

[14] Garbow, B. J., et al., Matrix Eigensystem Routines-EISPACK Guide Extension. Lecture Notes in Computer Science, Vol. 51. Springer-Verlag, New York/Heidelberg/Berlin, 1977.

[15] Smith, B. T., et al., Matrix Eigensystem Routines-EISPACK Guide, Second Edition. Lecture Notes in Computer Science, Vol. 6. Springer-Verlag, New York/Heidelberg/Berlin, 1976.

[16] Sparks, D. N. and Todd, A. D., Algorithm AS 60: Latent Roots and Vectors of a Symmetric Matrix. Applied Statistics, Vol. 22 (1973), pp. 260-265. Corrigendum: Applied Statistics, Vol. 23 (1974), pp. 101-102.

[17] Wilkinson, J. H. and Reinsch, C., editors, Handbook for Automatic Computation, Volume II, Linear Algebra (Springer-Verlag, New York/Heidelberg/Berlin, 1971).

[18] Waxler, R. M., Weir, C. E., and H. W. Schamp, Jr., Effect of Pressure and Temperature Upon the Optical Dispersion of Benzene, Carbon Tetrachloride and Water, J. Res. Nat'l. Bureau of Standards-A. Physics and Chemistry, 68A, No. 5, 489-498, 1964.

\section{Part III: Fitting Functions of Three or More Arguments}

\section{Introduction}

The first two papers in this series (Parts I and II) dealt with ordinary curve and surface fitting, i.e., with the fitting of functions of one or two arguments. In the latter case, it was assumed that the data were in the form of a two-way table with no cells missing. Similarly, we will assume in this paper, that each value of the function to be fitted is associated with a combination of the levels of three or more arguments, all combinations being present, and each one being associated with a single value of the function. In other words, we assume a "complete factorial" with no replications per cell. Of course, if one or more cells contain more than a single observation, one can substitute the average for these replicates. For purposes of empirical fitting, this should be quite acceptable, provided the precision of the single observations is satisfactory.

We present the method in terms of a single example, a function of three arguments. Generalization to functions of more than three arguments should be self-evident. However, the method may become cumbersome, and is not recommended as a first choice in these cases. 


\section{Illustration: Fitting the $F$ table}

Table 1 is a portion of the table of critical values of the $F$ distribution for the levels of significance $\mathbf{P}$, of $25,10,5$, and 1 percent, and for degrees of freedom, both in the numerator and in the denominator, of 4,6 , 60,120 , and $\infty$. The table, taken from ref. [1], has 100 "observations," but covers an infinite range of both sets of degrees of freedom, $\nu_{1}$ and $\nu_{2}$. We fully intend the empirical fit to be acceptable over this doublyinfinite range, and for all values of $P$ between 1 and 25 percent.

TABLE 1. F-Table. Data for Fit

\begin{tabular}{|c|c|c|c|c|c|}
\hline \multirow{7}{*}{$\begin{array}{r}\nu_{1} \\
4\end{array}$} & \multirow{3}{*}{$\begin{array}{r}\nu_{2} \\
4\end{array}$} & \multicolumn{4}{|c|}{$\begin{array}{c}P \text {-Level of Significance, in percent } \\
P\end{array}$} \\
\hline & & 25 & 10 & 5 & 1 \\
\hline & & 2.06 & 4.11 & 6.39 & 15.98 \\
\hline & 6 & 1.79 & 3.18 & 4.53 & 9.15 \\
\hline & 60 & 1.38 & 2.04 & 2.53 & 3.65 \\
\hline & 120 & 1.37 & 1.99 & 2.45 & 3.48 \\
\hline & $\infty$ & 1.35 & 1.94 & 2.37 & 3.32 \\
\hline \multirow[t]{5}{*}{6} & 4 & 2.08 & 4.01 & 6.16 & 15.21 \\
\hline & 6 & 1.78 & 3.05 & 4.28 & 8.47 \\
\hline & 60 & 1.35 & 1.87 & 2.25 & 3.12 \\
\hline & 120 & 1.33 & 1.82 & 2.17 & 2.96 \\
\hline & $\infty$ & 1.31 & 1.77 & 2.10 & 2.80 \\
\hline \multirow[t]{5}{*}{60} & 4 & 2.08 & 3.79 & 5.69 & 13.65 \\
\hline & 6 & 1.74 & 2.76 & 3.74 & 7.06 \\
\hline & 60 & 1.19 & 1.40 & 1.53 & 1.84 \\
\hline & 120 & 1.16 & 1.32 & 1.43 & 1.66 \\
\hline & $\infty$ & 1.12 & 1.24 & 1.32 & 1.47 \\
\hline \multirow[t]{5}{*}{120} & 4 & 2.08 & 3.78 & 5.66 & 13.56 \\
\hline & 6 & 1.74 & 2.74 & 3.70 & 6.97 \\
\hline & 60 & 1.17 & 1.35 & 1.47 & 1.73 \\
\hline & 120 & 1.13 & 1.26 & 1.35 & 1.53 \\
\hline & $\infty$ & 1.08 & 1.17 & 1.22 & 1.32 \\
\hline \multirow[t]{5}{*}{$\infty$} & 4 & 2.08 & 3.76 & 5.63 & 13.46 \\
\hline & 6 & 1.74 & 2.72 & 3.67 & 6.88 \\
\hline & 60 & 1.15 & 1.29 & 1.39 & 1.60 \\
\hline & 120 & 1.10 & 1.19 & 1.25 & 1.38 \\
\hline & $\infty$ & 1.00 & 1.00 & 1.00 & 1.00 \\
\hline
\end{tabular}

\section{Approach}

The method is simply stated. First, we combine two of the three factors, in this case $v_{1}$ and $v_{2}$, pretending it to be, for the time being, a single factor. We then apply the SVD (singular value decomposition) to the twoway table thus obtained. Each eigenvector will be a function of either $P$ or of the combination of a particular $v_{1}$ and a particular $v_{2}$. This latter type of eigenvector is then entered into a two-way table, as a function of $v_{1}$ and $v_{2}$. This two-way table is, itself, subjected to a SVD, with resulting eigenvectors that are functions of $v_{1}$ or $v_{2}$, taken singly. The problem is thus reduced to the fitting of a number of curves (functions of a single argument).

\section{Details of the fitting process}

\section{First step: $S V D$ of $25 \times 4$ table.}

The 25 rows are the combinations of the five levels of $v_{1}$ and the five levels of $v_{2}$; the four columns represent the four levels of the factor $P$ (see table 1). 
The SVD of this table, carried out to three terms, is represented by ${ }^{1}$

$$
y_{i t}=\theta_{1} u_{1 i} v_{1 t}+\theta_{2} u_{2 i} v_{2 t}+\theta_{3} u_{3 i} v_{3 t}
$$

with the values (see Parts I and II for notation and terminology):

$$
\begin{aligned}
\sum_{i \text { i }} y_{i t}^{2} & =1939.001 \\
\theta_{1} & =43.70505824 \\
\theta_{2} & =5.34641595 \\
\theta_{3} & =.53288922
\end{aligned}
$$

From these values we derive:

$$
\sum_{i t}\left(d_{i t}\right)_{3}^{2}=7.495692 \times 10^{-4}
$$

which gives an average square residual per observation of:

$$
\frac{7.495692 \times 10^{-4}}{5 \times 5 \times 4}=7.495692 \times 10^{-6}=(.00274)^{2}
$$

Thus, the fit will be good to approximately 3 units in the third place, provided that all the eigenvectors are fitted to an equivalent degree of approximation.

\section{Second step: Fitting the $v$ vectors}

All $v$ vectors are functions of a single variable, $P$, as shown in table 2 . They are readily fitted by the methods of Part 1, with the results shown in Table 3.

TABLE 2. $v$-Vectors as Functions of $P$

\begin{tabular}{rrrr}
\hline \hline$P$ & $v_{1}$ & $v_{2}$ & $v_{3}$ \\
\hline 25 & 0.161663 & 0.567164 & 0.750537 \\
10 & .275861 & .537558 & -.155183 \\
5 & .395108 & .412703 & -.613514 \\
1 & .861193 & -.468007 & .190293 \\
\hline
\end{tabular}

TABLE 3. Fit of v-Vectors

$$
\begin{gathered}
\text { a) MFP Fits } \\
y=y_{0}+A\left(x-x_{0}\right)\left|x-x_{0}\right|^{B-1} \\
\text { where } x=P ; y=v
\end{gathered}
$$

\begin{tabular}{ccccc} 
Vector & $x_{0}$ & $y_{0}$ & $A$ & $B$ \\
\hline$v_{1}$ & 0.104080 & -0.102867 & 0.923714 & -0.388978 \\
$v_{2}$ & -6.628102 & .568912 & -9496.645 & -4.489745 \\
\hline
\end{tabular}

b) QFP Fit

$$
\begin{gathered}
y=\mathrm{a}+\mathrm{b}\left(\frac{\mathrm{x}^{\mathrm{a}}-1}{\alpha}\right)+\mathrm{c}\left(\frac{\mathrm{x}^{\mathrm{e}}-1}{\alpha}\right)^{2} \\
\text { where } x=\mathrm{P} ; \mathrm{y}=\mathrm{v}
\end{gathered}
$$

\begin{tabular}{cccccc} 
Vector & $\alpha$ & $a$ & $b$ & $c$ & 0.931911 \\
\hline$v_{3}$ & -0.237709 & 0.190293 & -1.847334 & 0.96 \\
\hline
\end{tabular}

(1) The critical $F$ values are temporarily represented by the symbol $y_{i t}$. 
3. Third Step: SVD of the u vectors

Each $u$ vector is a function of $v_{1}$ and $v_{2}$ as shown by tables 4,5 , and 6 .

To avoid confusion, we will denote the eigenvectors resulting from the SVD of each $u$ vector by the sym. bols $A_{j}$ and $B_{k}$ for $u_{1}, C_{j}$ and $D_{k}$ for $u_{2}$, and $E_{j}$ and $G_{k}$ for $u_{3}$. We find that, to obtain sufficient precision, the SVD for $u_{1}$, requires three terms, while for $u_{2}$ and $u_{3}$ two terms suffice; thus: ${ }^{2}$

$$
\begin{aligned}
& u_{1}=T_{11} A_{1 j} B_{1 k}+T_{12} A_{2 j} B_{2 k}+T_{13} A_{3 j} B_{3 k} \\
& u_{2}=T_{21} C_{1 j} D_{1 k}+T_{22} C_{2 j} D_{2 k} \\
& u_{3}=T_{31} E_{1 j} G_{1 k}+T_{32} E_{2 j} G_{2 k}
\end{aligned}
$$

TABLE 4. $u_{1}-$ Vector as a Function of $\nu_{1}$ and $\nu_{2}$

\begin{tabular}{rrrrrr}
\hline$\nu_{2} \nu_{1}$ & 4 & 6 & 60 & 120 & $\infty$ \\
4 & 0.406210 & 0.388401 & 0.352024 & 0.349916 & 0.347548 \\
6 & .247943 & .231426 & .196782 & .194521 & .192350 \\
60 & .112774 & .098616 & .063326 & .060227 & .056489 \\
120 & .108349 & .094350 & .058259 & .054485 & .050072 \\
$\infty$ & .104083 & .090175 & .052868 & .048419 & .038755 \\
\hline
\end{tabular}

TABLE 5. $u_{2}$-Vector as a Function of $\nu_{1}$ and $\nu_{2}$

\begin{tabular}{rrrrrr}
$\nu_{2} \nu_{1}$ & 4 & 6 & \multicolumn{1}{c}{60} & \multicolumn{1}{c}{120} & $\infty$ \\
\hline 4 & -.273800 & -.232084 & -.153928 & -.149371 & -.144944 \\
6 & .058346 & .084441 & .132782 & .135561 & .139113 \\
60 & .227296 & .231801 & .224040 & .221888 & .218938 \\
120 & .229914 & .232483 & .220851 & .216840 & .212030 \\
$\infty$ & .230595 & .233936 & .216705 & .210834 & .196284 \\
\hline
\end{tabular}

TABLE 6. $u_{3}$.Vector as a Function of $\nu_{1}$ and $\nu_{2}$

\begin{tabular}{rrrrrr}
\hline$\nu_{2} \nu_{1}$ & 4 & 6 & 60 & 120 & $\infty$ \\
\hline 4 & 0.054105 & 0.101229 & 0.149334 & 0.154646 & 0.159300 \\
6 & -.352902 & -.284130 & -.137823 & -.118086 & -.109860 \\
60 & -.259816 & -.119461 & .163908 & .180098 & .215083 \\
120 & -.227942 & -.098102 & .195804 & .216706 & .256403 \\
$\infty$ & -.206583 & -.088254 & .221559 & .247171 & .323018 \\
\hline
\end{tabular}

4. Fourth Step: Fitting the vectors $A, B, C, D, E$, and $G$.

The vectors $A, C$, and $E$ are functions of $\nu_{1}$ only, while $B, D$, and $G$ are functions of $\nu_{2}$ only, as shown in table 7. Again, we use the methods of $P$ art $I$ to fit these vectors to their corresponding arguments, with the results shown in table 8 .

\section{Fifth step: Fit of $F$ as a function of $P, \nu_{1}$, and $\nu_{2}$.}

By substituting for $u_{1}, u_{2}$, and $u_{3}$ in eq. (1), their expressions as given by eqs (6), (7), and (8), one readily obtains an expression for $y_{i s}$ as a function of quantities that are either constants (the $\theta$ and the $T$ ), or functions of a single argument $\left(P, \nu_{1}\right.$, and $\left.\nu_{2}\right)$. Since the latter have all already been fitted in terms of their respective arguments, the problem is solved, except for the routine multiplications and additions involved in eqs (1), (6), (7), and (8). A program can readily be written to obtain the value of $y_{i t}$, that is, of $F$, for any $\nu_{1}, \nu_{2}$, and $P$, using eqs (1), (6), (7), (8) and the MFP or QFP fits shown in Tables 3 and 8.

\footnotetext{
The square roots of the eigenvalues are represented by the letter $T$, to avoid confusion with the $\theta$ of eq. (1)
} 
TABLE 7. SVD Vectors as Functions of $\nu_{1}$ or $\nu_{2}$ (Eqs. (6), (7), (8))

\begin{tabular}{rccccccc}
\hline \hline$\nu_{1}$ & $\mathrm{~A}_{1}$ & \multicolumn{1}{c}{$\mathrm{A}_{2}$} & \multicolumn{1}{c}{$\mathrm{A}_{3}$} & \multicolumn{1}{c}{$\mathrm{C}_{1}$} & $\mathrm{C}_{2}$ & \multicolumn{1}{c}{$\mathrm{E}_{1}$} & \multicolumn{1}{c}{$\mathrm{E}_{2}$} \\
\hline 4 & .828136 & -.362993 & -.216246 & -.436484 & -.712491 & .220226 & .407258 \\
6 & .479297 & .070208 & .361271 & .243432 & -.673184 & .053854 & -.898992 \\
60 & .179612 & .482627 & .431289 & .506592 & -.166174 & .511488 & -.141436 \\
120 & .168194 & .520863 & .266325 & .501735 & -.105627 & .554120 & -.025071 \\
$\infty$ & .154640 & .599231 & -.752183 & .491779 & -.020205 & .616385 & .072944 \\
& & & & & & & \\
$\nu_{2}$ & $\mathrm{~B}_{1}$ & \multicolumn{1}{c}{$\mathrm{B}_{2}$} & $\mathrm{~B}_{3}$ & $\mathrm{D}_{1}$ & $\mathrm{D}_{2}$ & $\mathrm{G}_{1}$ & $\mathrm{G}_{2}$ \\
\hline 4 & .511101 & .609203 & .238929 & .481716 & .687371 & -.473303 & .661606 \\
6 & .481313 & .367238 & -.143676 & .475017 & .314111 & -.195838 & .558564 \\
60 & .416240 & -.301559 & -.508432 & .434081 & -.344513 & .426098 & .311762 \\
120 & .411520 & -.379435 & -.342844 & .426716 & -.377055 & .471649 & .281945 \\
$\infty$ & .405595 & -.509016 & .739069 & .414481 & -.409871 & .577595 & .271280 \\
\hline
\end{tabular}

TABLE 8. Fit of Vectors of Table 7

\begin{tabular}{|c|c|c|c|c|}
\hline \multirow[b]{2}{*}{ Vector } & \multicolumn{3}{|c|}{$\begin{array}{c}\text { a) MFP Fits } \\
\begin{array}{c}y=y_{0}+A\left(x-x_{0}\right)\left|x-x_{0}\right|^{B-1} \\
\text { where } x=\nu_{1} \text { or } \nu_{2} ; y=\text { vector fitted }\end{array}\end{array}$} & \multirow[b]{2}{*}{$B$} \\
\hline & $x_{0}$ & $y_{0}$ & $A$ & \\
\hline$A_{1}$ & 2.333242 & .154595 & 1.080698 & -0.925484 \\
\hline$A_{2}$ & 3.160494 & .609721 & -.893793 & -.483655 \\
\hline$B_{1}$ & -.251639 & .405481 & .366518 & -.859647 \\
\hline$B_{2}$ & -.228726 & -.518182 & 2.767959 & -.622996 \\
\hline$D_{1}$ & -9.389754 & .414477 & .470463 & -.749928 \\
\hline$D_{2}$ & -.114913 & -.409594 & 4.845992 & -1.050171 \\
\hline$G_{1}$ & .678625 & .581829 & -2.303516 & -.650175 \\
\hline$G_{2}$ & -1.463892 & .269022 & 2.026138 & -.966873 \\
\hline
\end{tabular}

b) QFP Fits

$$
\begin{gathered}
y=a+b\left(\frac{x^{a}-1}{\alpha}\right)+c\left(\frac{x^{a}-1}{\alpha}\right)^{2} \\
\text { where } x=\nu_{1} ; y=\text { vector fitted }
\end{gathered}
$$

\begin{tabular}{crrrr} 
Vector & \multicolumn{1}{c}{$\alpha$} & \multicolumn{1}{c}{$a$} & \multicolumn{1}{c}{$c$} \\
\hline$A_{3}$ & -.428299 & -6.868927 & 9.408257 & -2.907025 \\
$B_{3}$ & -.325922 & 3.754828 & -4.383540 & 1.108433 \\
$C_{1}$ & -1.196614 & -34.856565 & 87.329516 & -53.883595 \\
$C_{2}$ & -.830262 & 3.094441 & -9.025929 & 5.346705 \\
$E_{1}$ & -1.010583 & 15.626475 & -37.446675 & 22.511319 \\
$E_{2}$ & -1.126615 & 93.240278 & -235.519021 & 147.086636 \\
\hline
\end{tabular}

\section{Results}

Table 9 shows the fitted value for each entry of table 1. As expected, the fit is very good. The sum of squares of the residuals for 100 values is 0.0109 . This gives a root mean square deviation per value of 0.0104 .

\section{Interpolation}

We mentioned earlier that the final fit should be adequate not only in reproducing the values of the original table, but also as an interpolation formula. In table 10, a comparison is made between values of $F$ as given by the Biometrika Tables, and those given by our empirical fit, for combination of $P, \nu_{1}$, and $\nu_{2}$ not 
included in table 1 (the basis for our formula). Note, in particular, the values for $P=2.5$ percent, a level that was totally absent from table 1 .

The sum of squares of residuals for these 80 values is 0.01812 . Thus, the root mean square deviation per value is 0.048 . Interpolation would of course be better if a larger table of $F$ values had been used for the fitting process.

TABLE 9. F Table-Fitted Values

\begin{tabular}{|c|c|c|c|c|c|}
\hline \multirow[b]{2}{*}{$\nu_{1}$} & \multirow[b]{2}{*}{$\nu_{2}$} & \multicolumn{4}{|c|}{$\mathrm{P}$} \\
\hline & & 25 & 10 & 5 & 1 \\
\hline \multirow[t]{5}{*}{4} & 4 & 2.07 & 4.11 & 6.40 & 15.98 \\
\hline & 6 & 1.78 & 3.18 & 4.52 & 9.15 \\
\hline & 60 & 1.39 & 2.04 & 2.54 & 3.66 \\
\hline & 120 & 1.37 & 1.98 & 2.45 & 3.47 \\
\hline & $\infty$ & 1.36 & 1.95 & 2.39 & 3.32 \\
\hline \multirow[t]{5}{*}{6} & 4 & 2.07 & 4.00 & 6.16 & 15.21 \\
\hline & 6 & 1.79 & 3.07 & 4.29 & 8.47 \\
\hline & 60 & 1.35 & 1.87 & 2.26 & 3.12 \\
\hline & 120 & 1.32 & 1.80 & 2.16 & 2.95 \\
\hline & $\infty$ & 1.31 & 1.76 & 2.10 & 2.82 \\
\hline \multirow[t]{5}{*}{60} & 4 & 2.07 & 3.78 & 5.69 & 13.66 \\
\hline & 6 & 1.75 & 2.77 & 3.74 & 7.06 \\
\hline & 60 & 1.19 & 1.40 & 1.54 & 1.84 \\
\hline & 120 & 1.15 & 1.32 & 1.42 & 1.65 \\
\hline & $\infty$ & 1.11 & 1.23 & 1.30 & 1.47 \\
\hline \multirow[t]{5}{*}{120} & 4 & 2.08 & 3.78 & 5.66 & 13.56 \\
\hline & 6 & 1.74 & 2.74 & 3.70 & 6.96 \\
\hline & 60 & 1.17 & 1.35 & 1.47 & 1.73 \\
\hline & 120 & 1.13 & 1.26 & 1.35 & 1.53 \\
\hline & $\infty$ & 1.08 & 1.16 & 1.21 & 1.31 \\
\hline \multirow[t]{5}{*}{$\infty$} & 4 & 2.09 & 3.77 & 5.64 & 13.46 \\
\hline & 6 & 1.73 & 2.72 & 3.66 & 6.89 \\
\hline & 60 & 1.15 & 1.29 & 1.38 & 1.60 \\
\hline & 120 & 1.10 & 1.18 & 1.23 & 1.36 \\
\hline & $\infty$ & 1.01 & 1.02 & 1.00 & .95 \\
\hline
\end{tabular}

TABLE 10. F Table-Interpolated Values ${ }^{\circ}$

\begin{tabular}{|c|c|c|c|c|c|c|c|c|c|c|c|}
\hline \multirow{2}{*}{\multicolumn{2}{|c|}{$P(\%)$}} & \multicolumn{10}{|c|}{$P$} \\
\hline & & \multicolumn{2}{|c|}{25} & \multicolumn{2}{|c|}{10} & \multicolumn{2}{|c|}{5} & \multicolumn{2}{|c|}{2.5} & \multicolumn{2}{|c|}{1} \\
\hline$\nu_{1}$ & $\nu_{2}$ & Tab. & Fit & Tab. & Fit & Tab. & Fit & Tab. & Fit & Tab. & Fit \\
\hline \multirow[t]{4}{*}{5} & 5 & 1.89 & 1.87 & 3.45 & 3.41 & 5.05 & 4.99 & 7.15 & 7.06 & 10.97 & 10.93 \\
\hline & 15 & 1.49 & 1.53 & 2.27 & 2.33 & 2.90 & 2.98 & 3.58 & 3.59 & 4.56 & 4.61 \\
\hline & 30 & 1.41 & 1.42 & 2.05 & 2.07 & 2.53 & 2.57 & 3.03 & 3.00 & 3.70 & 3.73 \\
\hline & 40 & 1.39 & 1.39 & 2.00 & 2.00 & 2.45 & 2.47 & 2.90 & 2.87 & 3.51 & 3.53 \\
\hline \multirow[t]{4}{*}{15} & 5 & 1.89 & 1.86 & 3.24 & 3.20 & 4.62 & 4.57 & 6.43 & 6.34 & 9.72 & 9.69 \\
\hline & 15 & 1.43 & 1.48 & 1.97 & 2.06 & 2.40 & 2.51 & 2.86 & 2.89 & 3.52 & 3.57 \\
\hline & 30 & 1.32 & 1.34 & 1.72 & 1.76 & 2.01 & 2.06 & 2.31 & 2.28 & 2.70 & 2.72 \\
\hline & 40 & 1.30 & 1.31 & 1.66 & 1.68 & 1.92 & 1.95 & 2.18 & 2.14 & 2.52 & 2.53 \\
\hline \multirow[t]{4}{*}{30} & 5 & 1.88 & 1.85 & 3.17 & 3.14 & 4.50 & 4.44 & 6.23 & 6.13 & 9.38 & 9.34 \\
\hline & 15 & 1.40 & 1.45 & 1.87 & 1.96 & 2.25 & 2.35 & 2.64 & 2.67 & 3.21 & 3.26 \\
\hline & 30 & 1.28 & 1.30 & 1.61 & 1.64 & 1.84 & 1.89 & 2.07 & 2.05 & 2.39 & 2.40 \\
\hline & 40 & 1.25 & 1.27 & 1.54 & 1.56 & 1.74 & 1.77 & 1.94 & 1.90 & 2.20 & 2.21 \\
\hline \multirow[t]{4}{*}{40} & 5 & 1.88 & 1.85 & 3.16 & 3.12 & 4.46 & 4.40 & 6.18 & 6.07 & 9.29 & 9.25 \\
\hline & 15 & 1.39 & 1.43 & 1.85 & 1.93 & 2.20 & 2.30 & 2.59 & 2.60 & 3.13 & 3.17 \\
\hline & 30 & 1.27 & 1.29 & 1.57 & 1.61 & 1.79 & 1.84 & 2.01 & 1.98 & 2.30 & 2.32 \\
\hline & 40 & 1.24 & 1.25 & 1.51 & 1.53 & 1.69 & 1.72 & 1.88 & 1.83 & 2.11 & 2.12 \\
\hline
\end{tabular}

- Tab. = tabulated, from [1]

Fit $=$ fitted by the procedure of this paper. 
The total number of parameters is the sum of 10 (one for each $\theta$ or $T$ ), and $4 \times 17$ (four for each of the three eigenvectors $v$, and four for each of the 14 vectors occuring in eqs (5), (6), and (7)); i.e., 78. As mentioned in Part II, this number can be somewhat reduced through algebraic manipulation, but this is unnecessary for a fitting process carried out on a programmable calculator or on a computer.

We finally repeat our previous assertion (see also Part II) that these 78 parameters fit not only a table of 100 observations (Table 1), (which would be a waste of time) but actually any $F$ value, for $P$ between 1 and 25 percent, and for $\nu_{1}$ and $\nu_{2}$ between 4 and $\infty$.

\section{Conclusion for Part III}

Through repeated application of the procedure given in Part II, it is possible to fit functions of more than two arguments, provided the data appear as a complete factorial. This is accomplished by first combining all combinations of two or more factors into one factor until a two-way table is obtained. The parameter vectors of the SVD of this table are then expressed as two-way tables themselves and further SVD's are carried out. The procedure is simple in principle but can become quite cumbersome in practice. It is not recommended for functions of more than three arguments, unless no other appropriate fitting procedure is available.

\section{References}

[1] Pearson, E. S., and H. O. Hartley, Editors, Biometrika Tables for Statisticians, (Cambridge University Press, London, 1970). 